\title{
PERCEPCIÓN DE LOS EXPERTOS DE LA NECESIDAD DE LA FORMACIÓN DEL PROFESORADO EN CONVIVENCIA
}

\section{PERCEPTIONS EXPERTS NEED FOR TEACHER TRAINING IN COEXISTENCE}

\author{
Antonia Penalva López \\ $M^{a}$ Ángeles Hernández Prados \\ Catalina Guerrero Romera \\ Universidad de Murcia
}

Fecha de recepción: 12 de junio de 2013

Fecha de aceptación: 08 de diciembre de 2013

Fecha de publicación: 30 de junio de 2014

\section{RESUMEN}

Uno de los desafíos propios de la institución escolar, es propiciar un clima favorable a la convivencia, que facilite el desarrollo instructivo y pedagógico, que en muchas ocasiones se ve interrumpido y alterado por conductas violentas de diversa índole que suelen ser gestionadas principalmente por los docentes. Sin embargo, para que esta gestión sea eficaz, el profesorado debe conocer y poner en práctica una serie de recursos y estrategias de gestión, de las cuales en muchas ocasiones carece. De ahí la necesidad de centrar nuestro estudio en conocer que necesidades formativas tienen los docentes según la percepción de expertos en convivencia escolar. Para ello se elaboró un panel de expertos que consta de siete cuestiones abiertas, enviadas a un total de 30 expertos y obteniendo una participación final del $40 \%$. Entre sus resultados cabe destacar el (75\%) de expertos que consideran necesario basar la formación docente en competencias cognitivas, y conductuales $(91,66 \%)$; conocer las medidas educativas que se llevan a cabo en el centro $(91,66 \%)$ y las estrategias de intervención $(91,66 \%)$; además de las causas que provocan los conflictos escolares como los factores escolares $(91,66 \%)$ y familiares $(66,66 \%)$.

Palabras claves: Expertos; Formación; Profesorado; Convivencia.

\begin{abstract}
One of the challenges of the school is to foster a climate conducive to coexistence, to provide instructive and educational development, which often is interrupted and altered by violent behavior of various kinds which are usually managed mainly by teachers. However, for this management is effective, teachers must know and implement a range of resources and strategies for management, which often lacks. Hence the need to focus our study revealed that teachers have training needs as perceived by experts in school life. For this, we create a panel of experts consisting of 7 open questions, sent to a total of 30 experts and obtaining a stake of $40 \%$. The results included to highlight the $(75 \%)$ of experts considered necessary to base teacher training in cognitive, and behavioral (91.66\%); meet educational measures are carried out in the center (91.66\%) and intervention strategies (91.66\%), in addition to the causes of school conflicts as school factors (91.66\%) and relatives (66.66\%).
\end{abstract}

Key words: Expert; Training; Teaching; Fellowship. 
Percepción de los expertos de la necesidad de la formación del profesorado en convivencia

\section{NECESIDAD FORMATIVA DE LOS DOCENTES EN CONVIVENCIA.}

Las conductas agresivas en los centros educativos han dejado de percibirse como el resultado de la precocidad de los educandos, hasta llegar a reconocer la dimensión del problema, que afecta, no sólo al funcionamiento de las instituciones sino al desarrollo integral y académico de los alumnos. No cabe duda que esta situación se contrapone con los deseos e intenciones de la comunidad educativa, de favorecer la convivencia escolar, entendida como la capacidad de vivir juntos en el mismo contexto, respetando los derechos de todos y los sentimientos y emociones individuales de cada sujeto (Ortega, 2006), reivindicando que se inviertan esfuerzos de todo tipo en materializar todos los aprendizajes señalados por Delors (1996), pero enfatizando la enseñanza de aprender a convivir bajo los fundamentos y valores propios de una sociedad democrática.

Concebir los conflictos como enriquecimiento personal, útiles y valiosos para el alumnado si son gestionados constructivamente, promoviendo el razonamiento cognitivo y moral y unas relaciones más apacibles y propicias para una buena convivencia (Rodríguez, 2007), es algo poco frecuente en la comunidad educativa. Esto suscita el interés y la necesidad de aplicar una educación basada en valores de convivencia y preventiva de conductas antisociales (Ortega y del Rey, 2001), lo que ha dado lugar a la proliferación de múltiples programas de convivencia como (SAVE), (ANDAVE), Convivir es vivir, etc.

Las pretensiones iniciales de estas iniciativas de dar respuesta a la violencia entre iguales han ido evolucionando hasta trazar líneas de actuación orientadas a la promoción de las relaciones entre los miembros de la comunidad, de las habilidades socio-emocionales y la formación docente en convivencia (Uruñuela, 2012). Habitualmente estas iniciativas vienen asociadas a programas formativos, aunque sus insuficiencias han sido demostradas por el (ANPE, 2003), quienes revelan que el $(81,7 \%)$ de docentes reconocen no haber recibido formación en gestión de conflictos escolares. No obstante, el $(20,8 \%)$ afirman haberla recibido formación al respecto, calificándola de poco satisfactoria (Jares, 2006), reclamando aprendizajes basado en el diálogo con las victimas $(68,6 \%)$ y agresores $(65,7 \%)$, trabajo con padres $(55 \%$ y $61,9 \%)$ y reflexión con el alumnado sobre la temática (59,9\%) (Caurcel, 2005).

De estos datos denotamos la importancia que los propios docentes otorgan a su formación, y desde ahí se debe partir, pues es el educador quien posee la coyuntura necesaria para gestionar la convivencia y trasformar los conflictos a través de la mediación y la 
negociación, favoreciendo un clima basado en la tolerancia (Narejo y Salazar, 2002).

Partiendo del rol docente como mediador y gestor de la convivencia, los programas de formación deberían girar en torno a la concesión de estrategias de intervención, orientadas al desarrollo de actividades a nivel de aula y de centro, así como el trabajo curricular mediante el aprendizaje cooperativos donde se fomente el trabajo en grupo (Ortega et al, 2002).

\section{PANEL DE EXPERTOS}

Se trata de una técnica metodológica de investigación sociológica, a través de la cual se recoge y analiza información sobre un tema concreto, de un grupo de expertos, que nos facilitan datos que garantizan nuestra correcta toma de decisiones (Armas, 2007).

El panel de expertos se caracteriza por presentar una serie de peculiaridades que lo hacen diferente a los demás. Una de las características propias de esta técnica es el anonimato, existiendo total desconocimiento entre los miembros, de las identidades de los demás participantes, lo que permite la libre expresión de ideas y opiniones (Martínez, 2003), favoreciendo el enriquecimiento de contenidos de la investigación. Como toda técnica metodológica, requiere de un proceso de elaboración que se compone de cinco fases: definición de objetivos, selección de expertos, diseño desarrollo del panel y análisis y exposición de resultados (Astigarraga, 2007).

La selección de los participantes es una de las fases más importantes del diseño del panel, y en ella se debe tener en cuenta el número de participantes a los que va dirigido nuestro instrumento. Sin embargo este es un aspecto dubitativo, por las discrepancias en las apreciaciones ofrecidas por los expertos, sobre el número de participantes adecuado para esta técnica. Unos consideran que el número dependería tanto de los objetivos como de las condiciones de cada estudio, debiendo ser lo más amplio posible para permitir la diversidad y riqueza de opiniones (Fernández, 1995), no recomendando superar los 50 participantes (Landeta, 1999). Por el contrario, otros consideran que este dato es un exceso de muestra, y apuntan que el número adecuado oscilaría entre 10 y 30 componentes (Ruiz e Ispizua, 1989), aunque no debería ser inferior a 15 expertos por estudio (Martino, 1983) (Citado en Castillo, 2002). No obstante la Comisión Europea (2005) se atreve a establecer unos criterios de profesionalidad, independencia 
Percepción de los expertos de la necesidad de la formación del profesorado en convivencia

y capacidad de trabajo en equipo para organizar grupos de expertos no inferiores a 5 ni superiores a 10.

Actualmente esta técnica va dirigida principalmente a tres tipos de expertos: los especialistas con conocimientos, capacidades 0 experiencias identificadas con la temática del panel; los afectados, que a pesar de no poseer una formación específica se ven involucrados en el tema principal del panel; y los facilitadores que poseen la capacidad de incitar al grupo incorporando nuevas ideas y percepciones (Landeta, 1999). En esta ocasión la muestra de expertos de este trabajo se compone exclusivamente de especialistas en el campo temático objeto de estudio.

En lo que respecta al diseño del panel, este proceso debe fundamentarse en unos criterios estructurales, como el orden de las variables y la claridad en la redacción de las cuestiones, que como bien indica García (2003) es una condición bastante exigible en este tipo de instrumentos, consiguiendo finalmente la formulación de preguntas fáciles de comprender y necesarias para recoger la información deseada. Una vez diseñado el instrumento, da comienzo la etapa de desarrollo, con el envío de los paneles a los expertos, facilitando a la vez un período de tiempo para su cumplimentación y devolución. En ocasiones se contempla un recordatorio y un segundo periodo de envío del panel cumplimentado, en el caso de no haber obtenido un número de respuestas considerables por parte de los expertos.

El análisis y exposición de los resultados es una etapa caracterizada por el proceso de análisis, pues las investigaciones cualitativas requieren de tiempo, y dependiendo de la fase de análisis en la que se encuentren se requerirá más o menos. Según (ÁlvarezGayou, 2005) el análisis de datos debe ser consecuente con un orden, razón por la cual (Álvarez- Gayou, 2005; Miles y Humberman, 1994; Rubin y Rubin, 1995) citado en (Fernández, 2006), establecen la siguiente secuencia por la que se debe seguir todo análisis cualitativo:

1. Obtener información

2. Capturar, transcribir y ordenar información. Esta etapa del análisis facilitará la codificación de la información.

3. Codificar información por categorías.

4. Integrar la información, para reducir los datos obtenidos (Codificación):

a) Muestreo: Para la identificación de la información integrada se estableció un sistema de códigos a los cuestionarios recibidos.

b) Identificación de temas relevantes para la investigación. 
c) Construir sistema de códigos en función a las categorías establecidas previamente.

d) Marcar textos relevantes, estableciendo unidades de significación.

e) Construir modelos conceptuales, mediante la relación de las unidades de significación con el sistema de códigos y con la muestra participante.

A continuación se desarrolla cada una de estas etapas que se han proseguido para la elaboración y aplicación del panel de expertos de nuestra investigación.

\section{PANEL DE EXPERTOS "LA PERCEPCIÓN DEL PROFESORADO SOBRE LA CONVIVENCIA EN LOS CENTROS EDUCATIVOS"}

\subsection{Definición de Objetivos}

Con este instrumento se pretende conocer que necesidades formativas tienen los docentes según la percepción de expertos en convivencia escolar. Con el propósito de dar respuesta a este objetivo general se plantearon los siguientes objetivos específicos:

- Identificar los aspectos de una percepción positiva y negativa en educación primaria y secundaria.

- Enumerar los tipos de conflictos que pueden producirse en el aula o en el centro.

- Determinar qué factores son, según los expertos, los instigadores de conflictos escolares.

- Conocer que efectos o consecuencias tiene una convivencia con conflictos en los centros educativos.

- Señalar las medidas educativas de centro para atender la mejora de la convivencia escolar.

- Identificar que estrategias docentes consideran necesarias los expertos, para resolver los conflictos en el aula.

- Determinar las competencias cognitivas, conductuales y actitudinales en las que debería basarse la formación del profesorado para la convivencia.

\subsection{Diseño del panel}

Planteados los objetivos y establecidos los criterios de selección de la muestra, se procedió al diseño del panel de expertos. Esta fase se caracteriza por la propuesta de contenidos, el formato del instrumento, 
Percepción de los expertos de la necesidad de la formación del profesorado en convivencia

así como la formulación de cuestiones que deben perseguir los siguientes criterios:

- Una estructura ordenada por bloques temáticos, basada en los objetivos y variables de la investigación.

- Exposición clara y simplificada de los interrogantes.

- Determinación del número de interrogantes en proporción al número de objetivos planteados.

Finalmente el panel de expertos consta de 7 cuestiones abiertas relacionadas con cada uno de los objetivos específicos propuestos en este estudio.

\begin{tabular}{|c|c|c|}
\hline $\begin{array}{l}\text { OBJETIVO } \\
\text { GENERAL }\end{array}$ & OBJETIVO ESPECIFICO & PREGUNTA \\
\hline \multirow{7}{*}{$\begin{array}{c}\text { Conocer que } \\
\text { necesidades } \\
\text { formativas } \\
\text { tienen los } \\
\text { docentes } \\
\text { según la } \\
\text { percepción } \\
\text { de expertos } \\
\text { en } \\
\text { convivencia } \\
\text { escolar }\end{array}$} & $\begin{array}{l}\text { Identificar los aspectos de } \\
\text { una percepción positiva y } \\
\text { negativa en educación } \\
\text { primaria y secundaria. }\end{array}$ & $\begin{array}{l}\text { Señale aspectos de una percepción } \\
\text { positiva y negativa por parte del } \\
\text { profesorado sobre la convivencia en } \\
\text { centros de educación Primaria y } \\
\text { Secundaria. }\end{array}$ \\
\hline & $\begin{array}{l}\text { Enumerar los tipos de } \\
\text { conflictos que pueden } \\
\text { producirse en el aula o en el } \\
\text { centro. }\end{array}$ & $\begin{array}{l}\text { Enumera los tipos de conflictos que se } \\
\text { pueden producir en un aula y en un } \\
\text { centro. (Priorice sus respuestas según } \\
\text { su gravedad, de más grave a menos } \\
\text { grave). }\end{array}$ \\
\hline & $\begin{array}{l}\text { Determinar qué factores son, } \\
\text { según los expertos, los } \\
\text { instigadores de conflictos } \\
\text { escolares }\end{array}$ & $\begin{array}{l}\text { ¿Cuáles crees que son los factores } \\
\text { que provocan la aparición de } \\
\text { conflictos escolares? ¿ Cuál cree que } \\
\text { influye principalmente? }\end{array}$ \\
\hline & $\begin{array}{l}\text { Conocer que efectos o } \\
\text { consecuencias tiene una } \\
\text { convivencia con conflictos } \\
\text { en los centros educativos. }\end{array}$ & $\begin{array}{l}\text { ¿Cuáles cree que son los efectos o } \\
\text { consecuencia de la mala } \\
\text { convivencia en los centros } \\
\text { educativos? }\end{array}$ \\
\hline & $\begin{array}{l}\text { Señalar las medidas } \\
\text { educativas de centro para } \\
\text { atender la mejora de la } \\
\text { convivencia escolar. }\end{array}$ & $\begin{array}{l}\text { ¿Qué medidas educativas de centro } \\
\text { para atender la mejora de la } \\
\text { convivencia escolar conoce? } \\
\text { Ordénelas según su efectividad de } \\
\text { mayor a menor. }\end{array}$ \\
\hline & $\begin{array}{l}\text { Determinar las competencias } \\
\text { cognitivas, conductuales y } \\
\text { actitudinales en las que } \\
\text { debería basarse la formación } \\
\text { del profesorado para la } \\
\text { convivencia. }\end{array}$ & $\begin{array}{l}\text { En que competencias cognitivas, } \\
\text { conductuales y actitudinales debería } \\
\text { basarse la formación del profesorado } \\
\text { en cuanto a convivencia escolar? }\end{array}$ \\
\hline & $\begin{array}{l}\text { Identificar que estrategias } \\
\text { docentes consideran } \\
\text { necesarias los expertos, para } \\
\text { resolver los conflictos en el } \\
\text { aula. }\end{array}$ & $\begin{array}{l}\text { ¿Qué estrategias del profesorado } \\
\text { consideras necesarias para la } \\
\text { resolución de conflictos en el aula? } \\
\text { ¿Y en el centro? (Valora la más } \\
\text { adecuada) }\end{array}$ \\
\hline
\end{tabular}

Tabla 1. Cuestiones del panel de expertos 


\subsection{Selección de expertos}

La muestra está formada exclusivamente por especialistas en convivencia escolar, con el único propósito de recoger información relevante relativa a nuestra investigación. Concretamente los participantes deben contar con unos requisitos previamente establecidos en el estudio como criterios de selección: Ser docentes universitarios expertos y abalados por estudios, libros y publicaciones sobre convivencia y conflictos escolares; o expertos de observatorios de convivencia de cualquier comunidad autónoma cuya función principal es trabajar en pro de una mejora en la convivencia de nuestras instituciones educativas.

Finalmente se seleccionaron un total de 30 expertos, de los cuales el $60 \%$ pertenecían al género masculino y el $40 \%$ al género femenino. Además el $86,66 \%$ correspondía a docentes universitarios, el $10 \%$ a observatorios de convivencia y el $3,33 \%$ docentes de I.E.S. Todos los expertos pertenecían a instituciones de las diferentes comunidades autónomas, concretamente el $26,26 \%$ eran pertenecían a Andalucía y Murcia, el $20 \%$ a Castilla y León, el $10 \%$ a Valencia, el $6,66 \%$ a Galicia y el 3,33\% a Navarra, Cantabria e Islas Baleares.

\subsection{Desarrollo del panel}

Previo al envío del panel de expertos se localizaron los correos electrónicos de todos los expertos, mediante su búsqueda en Internet o en sus universidades de procedencia, o recurriendo a la vía telefónica. Una vez recogidos todos los datos pertinentes de cada participante, y habiendo enmarcado esta investigación en un trabajo de Tesis Doctoral vinculada al departamento de Teoría e Historia de la Educación de la universidad de Murcia, se procedió al envío de los paneles, que iban acompañados de un certificado oficial de la universidad desde donde se realiza la investigación, así como una solicitud que recogía una presentación personal del investigador, el objetivo principal del panel de expertos, el intervalo de tiempo para la devolución, etc.

Finalmente obtuvimos respuesta del $26,26 \%$ de la muestra principal, lo que nos hizo plantearnos la posibilidad de un segundo envío recordatorio. Trascurrido unas semanas, decidimos llevar a cabo una segunda ronda recordatorio, volviendo a remitir nuevamente el panel de expertos con la información oportuna, acentuando nuestro interés en su participación, pues ayudaría a enriquecer la investigación. En este segundo envío obtuvimos respuesta del $13,33 \%$. Finalmente obtuvimos una participación total del $40 \%$ de la muestra principal, compuesta por el $23,33 \%$ de género masculino y el $16,66 \%$ de género femenino. De este 
Percepción de los expertos de la necesidad de la formación del profesorado en convivencia

40\%, pertenecían a la Comunidad autónoma de Murcia el (16\%), Andalucía (16\%), la comunidad de Madrid el $(3,33 \%)$ y a Cantabria el $(3,33 \%)$. A continuación se muestra un cronograma en el que se detalla cada una de las etapas del desarrollo del panel.

\begin{tabular}{|c|c|c|c|c|c|c|c|c|}
\hline \multicolumn{9}{|c|}{ DESARROLLO DEL PANEL DE EXPERTOS } \\
\hline \multirow[t]{3}{*}{ ETAPA } & \multicolumn{7}{|c|}{ FECHAS } & RESULTADO \\
\hline & \multicolumn{7}{|c|}{ ENERO } & \\
\hline & $L$ & $M$ & Mi & $J$ & $\boldsymbol{V}$ & $S$ & $D$ & \\
\hline $\begin{array}{l}\text { Búsqueda de } \\
\text { datos }\end{array}$ & & 1 & 2 & 3 & 4 & 5 & 6 & $\begin{array}{l}\text { Nombre de los participantes, } \\
\text { institución a la que pertenecen, } \\
\text { estudios que los abalan, números de } \\
\text { teléfono y correos electrónicos }\end{array}$ \\
\hline \multirow[t]{2}{*}{$\begin{array}{l}1^{a} \text { Ronda de } \\
\text { envíos }\end{array}$} & 7 & 8 & 9 & 10 & 11 & 12 & 13 & Total de participantes 30 . \\
\hline & 14 & 15 & 16 & 17 & 18 & 19 & 20 & \\
\hline \multirow[t]{4}{*}{ Respuestas } & 21 & 22 & 23 & 24 & 25 & 26 & 27 & $\begin{array}{l}\text { Participación de la } 1^{a} \text { ronda de un } \\
26,26 \% \text {, de la muestra principal. }\end{array}$ \\
\hline & 28 & 29 & 30 & 31 & & & & \\
\hline & \multicolumn{7}{|c|}{ FEBRERO } & \\
\hline & & & & & 1 & 2 & 3 & \\
\hline \multirow[t]{2}{*}{$\begin{array}{l}2^{a} \\
\text { recordatorio }\end{array}$} & 4 & 5 & 6 & 7 & 8 & 9 & 10 & $\begin{array}{llr}\text { Total de } & \text { participantes } & 22, \\
\text { concretamente } & \text { el } 73,33 \% \text { de la } \\
\text { muestra. } & & \end{array}$ \\
\hline & 11 & 12 & 13 & 14 & 15 & 16 & 17 & \\
\hline \multirow[t]{2}{*}{ Respuestas } & 18 & 19 & 20 & 21 & 22 & 23 & 24 & $\begin{array}{l}\text { Participación de la } 2^{a} \text { ronda de un } \\
13,13 \% \text { de la muestra principal. }\end{array}$ \\
\hline & 25 & 26 & 27 & 28 & & & & \\
\hline \multicolumn{8}{|c|}{ Participación final } & $40 \%$ de la muestra principal \\
\hline
\end{tabular}

Tabla 2. Desarrollo Panel de Expertos

\subsection{Análisis de datos}

Una vez recibidos todos los paneles cumplimentados, se procedió al análisis de los mismos, para ello continuamos un proceso fragmentado en las siguientes etapas: Enumeración y codificación; Organización y transcripción; Categorización; Codificación y recuento; Exposición de resultados.

El análisis de la información comenzó con la enumeración y codificación de cada uno de los cuestionarios recibidos, con el propósito de salvaguardar el anonimato de cada uno de los participantes e identificar fácil y directamente de la información recibida con su autor de referencia (Anexo I: Tabla de codificación de los paneles). 
Se continuó posteriormente con la organización de la información por cuestiones. Para ello se elaboró un matriz de vaciado, que recogía el orden de las cuestiones, la categoría y subcategorias de cada cuestión, así como las respuestas aportadas por cada uno de los participantes, con su respectivo código identificatorios (Anexo I: Parrilla vaciado de información).

A continuación, se identificaron unidades de información por cada una de las cuestiones del panel de expertos elaborando un sistema de categorías que posteriormente nos permitiera cuantificar la información (Anexo II: Unidades de información). Una vez establecido el sistema de categorías se realizó una lectura, destacando los textos más relevantes, relacionados con el sistema de categorías, instaurando finalmente unidades de información. Esta información fue almacenada en una parrilla que relacionaba la categoría con su unidad de información recogida de cada panel. Finalmente se asignó un código a cada una de las categorías, y se sucedió con la cuantificación de datos. Para ello se elaboró una segunda parrilla que recogía la relación entre el sistema de categorías con sus respectivos códigos y unidades de información, y la cuantificación final. Una vez obtenidos los datos finales, se elaboró un informe recogiendo los resultados obtenidos del análisis de datos y que se muestra a continuación.

\section{RESULTADOS}

A continuación se exponen los datos obtenidos del análisis de contenidos, que nos ayudaran a dar respuesta a los objetivos planteados en el panel de expertos.

Una de las dimensiones recogidas en el panel es la determinación de aquellas competencias cognitivas, conductuales y actitudinales en las que se debería basar la formación del profesorado para la convivencia. De modo que, el $(75 \%)$ de expertos los que consideran necesario una formación docente centrada en competencias cognitivas, entendidas como aquel conjunto de conocimientos, habilidades y actitudes que el docente debe conocer y poner en práctica en su proceso de gestión (Vásquez, 2010). Concretamente pretenden una formación basada en el conocimiento de las causas del conflicto (75\%), como el contexto social, las características personales e individuales de los educandos, así como su realidad psicobiológica y socio-cultural.

Sin embargo el (58,33\%) entienden que las competencias cognitivas deben estar basadas en el conocimiento de las estrategias y 
Percepción de los expertos de la necesidad de la formación del profesorado en convivencia

métodos de gestión de la convivencia, fundamentalmente en adquirir un saber sobre mediación, aprendizaje cooperativo, desarrollo de habilidades sociales, toma de decisiones en equipo, y sobre las teorías educativas que orientan los procesos educativos.

El $(25 \%)$ de los expertos entienden que un mayor conocimiento y comprensión de la terminología y conceptos relacionados con la convivencia ayudarían al docente a realizar una gestión más eficaz y tan sólo el $(16,16 \%)$ consideran relevante incluir en la formación docente, la normativa establecida en los centros educativos, que regular la convivencia escolar, principalmente las funciones del tutor, y los deberes, derechos y obligaciones de cada uno de los agentes activos las instituciones recogidos en el reglamento del centro.

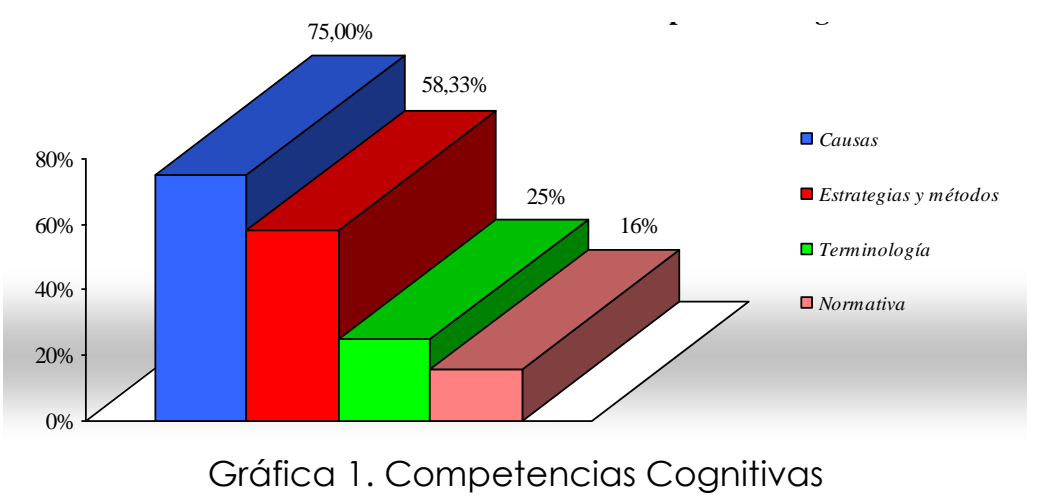

En lo que respecta a las competencias conductuales, centradas en el comportamiento de los docentes (Tobón, 2008), el $(91,66 \%)$ de los expertos discurren que la formación para la convivencia debe fundamentarse principalmente en su gestión e intervención, especialmente en la práctica de estrategias de gestión centralizadas en el trabajo en equipo a través de debates, intercambio de ideas, la dramatización y el uso de literatura infantil y juvenil, además del entrenamiento en el diálogo tanto grupal como individual y el manejo de las problemáticas de Bullying. No obstante, el $(16,66 \%)$ de la muestra consideran relevante centrarse en competencias conductuales orientadas a las normas y el $(8,33 \%)$ a los valores, esencialmente en la transversalidad en contenidos sobre ciudadanía evitando la transmisión de valores inadecuados. 


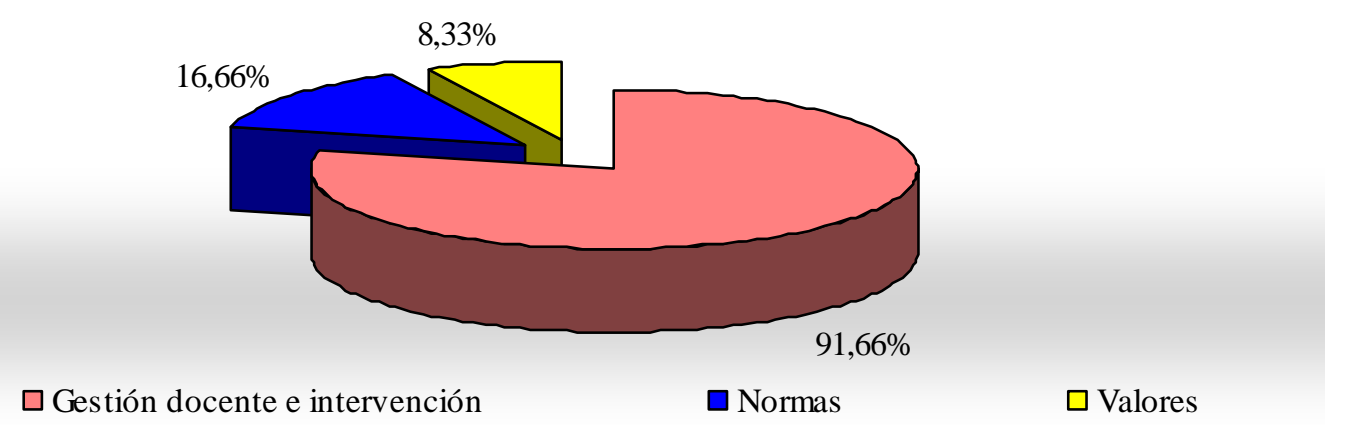

Gráfico 2. Competencias Conductuales

La opinión de los expertos respecto a la importancia de una formación centrada en actitudes relacionales fundamentadas en una percepción del conflicto como oportunidad de aprendizaje para una convivencia pacífica, especialmente centrada en las dimensiones de desarrollo humano, la empatía, capacidad de escucha activa, asertividad, dialogo, autoridad moral, acogida, desarrollo de habilidades sociales y de una actitud crítica $(66,66 \%)$. Sin embargo, el $41,66 \%$ estiman necesario orientar la formación hacia las actitudes educativas centralizadas hacia una actitud de implicación, creatividad, innovación y liderazgo, basadas en la sensibilización ante los problemas de convivencia, en la mediación y la percepción de los conflictos como oportunidad de aprendizaje.

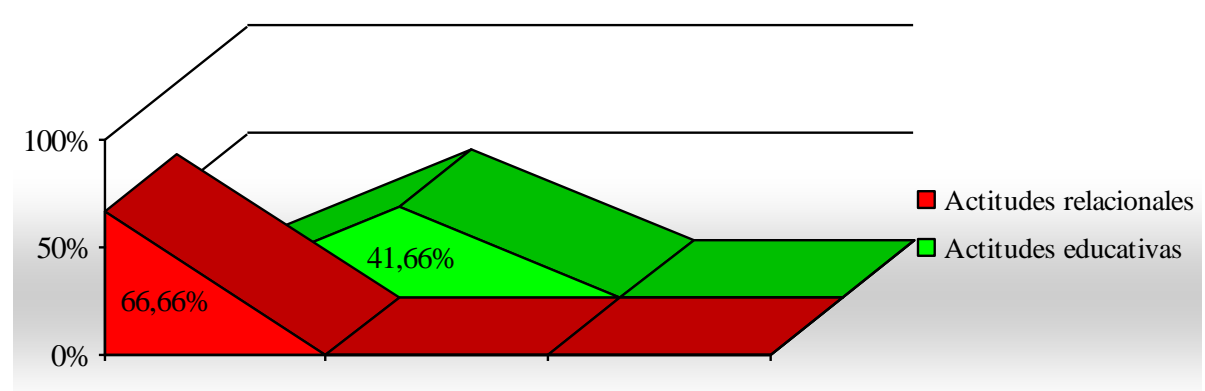

Gráfico 3. Competencias Actitudinales

El $91,66 \%$ de expertos conceden gran relevancia a las medidas educativas de centro, entendidas como todas aquellas acciones establecidas desde la propia institución para dar respuesta desde la prevención e intervención, a los problemas de convivencia, como los protocolo de acoso o Bullying, la elaboración y aplicación de planes de convivencia, el plan de acción tutorial, así como la elaboración del reglamento de régimen interno. Pese a este alto porcentaje, el 66,66\% de expertos consideran igualmente necesarias las medidas destinadas al docente, entendidas como aquellas acciones realizadas por el 
Percepción de los expertos de la necesidad de la formación del profesorado en convivencia

docente para la mejora de las relaciones interpersonales como la mediación escolar, establecimiento de putas de actuación comunes a todos los docentes, así como favorecer el apoyo del alumnado en la resolución de conflictos y poner en marcha actuaciones metodológicas concretas de aula.

No obstante el $50 \%$ consideran necesarias las medidas dirigidas al alumnado concebidas como toda acción donde el alumnado esté implicado como el establecimiento de normas a través de la negociación entre alumnos-profesor.

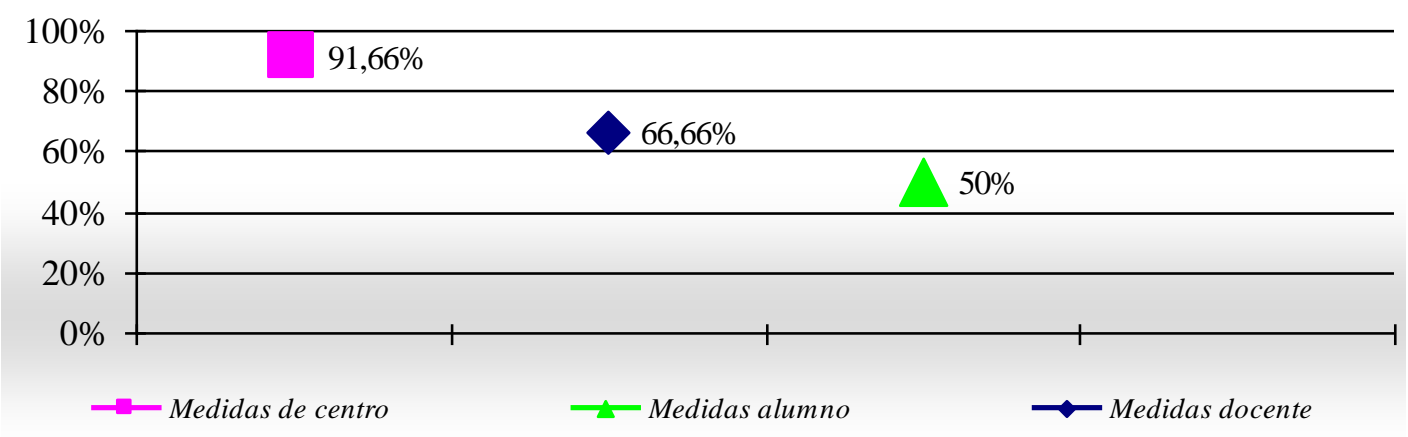

Gráfico 4. Medidas Educativas

Otra de las dimensiones de nuestra investigación estaba dirigida a la identificación de estrategias docentes para la gestión de conflictos, pudiendo comprobar que el $91,66 \%$ de la muestra consideran indispensables una formación en estrategias de intervención, especialmente centradas en estrategias metodológicas como el aprendizaje cooperativo grupos de calidad, dramatizaciones, rolplaying y la mediación escolar, así como el tratamiento de conflictos mediante terapias realistas, la comunicación de sentimientos y emociones, y el control tanto de las situaciones conflictivas como del alumnado disruptivo.

Por otro lado, el $83 \%$ de los expertos aluden a las estrategias preventivas para evitar futuros problemas en la convivencia, especialmente centradas en el establecimiento de una normativa de aula clara, así como una mayor formación del docente en estrategias y recursos pedagógicos como el aprendizaje de habilidades sociales y comunicativas, y nuevos métodos creativos de gestión de conflictos, que les ayuden no solo a una gestión más eficaz de la convivencia sino a favorecer la implicación tanto del alumnado como de las familias. 


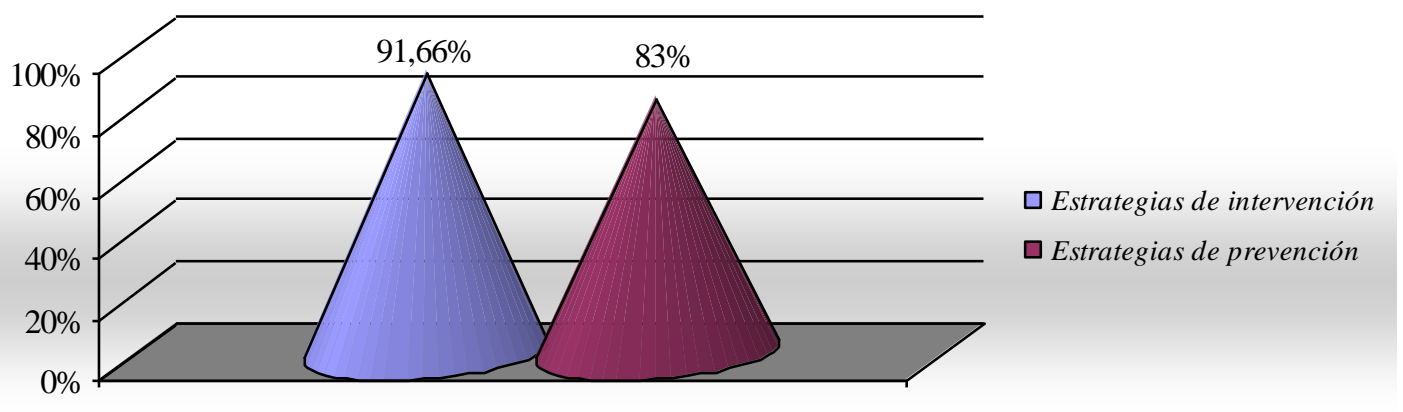

Gráfico 5. Estrategias del Profesorado

Con este estudio se pretendía identificar aquellos aspectos que los expertos consideraban positivos y negativos en educación primaria y secundaria. Sin embargo consideraron que tanto en primaría como en secundaría era preciso centrar la formación en la trasformación de aquellos aspectos dañinos para la convivencia y reforzar aquellos otros que eran favorecedores de una convivencia positiva. Concretamente, conceden alto grado de significación a la renovación de aspectos negativos de la percepción docente en primaria $41,66 \%$ referidos a comportamientos disruptivos y la implicación de la familia, el 33,33\% consideran necesario basar el cambio en la práctica docente, especialmente la percepción negativa que poseen del conflicto, así como las limitaciones funcionales de su práctica docente, centrada únicamente en funciones académicas. Sin embargo el $16,66 \%$ de expertos consideran necesario modificar aspectos como la carencia existente en la transmisión de una educación en valores, y solo el $(8,33 \%)$ en la normativa de centro que regula la convivencia.

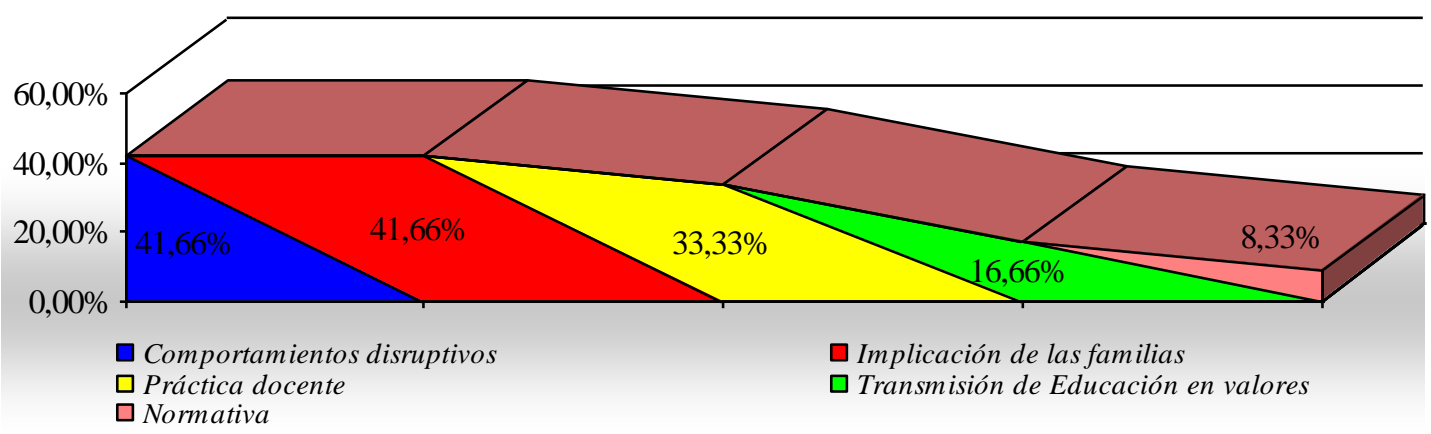

Gráfico 6. Aspectos Negativos en Primaria

Los expertos que consideran que en secundaría es necesario trasformar la autoridad y práctica docente (25\%) pretenden, principalmente, una transformación de la gestión basada en un modelo sancionador y punitivo a una gestión fundamentada en el modelo integrador donde predomina el dialogo asertivo y la negociación. Además $(16,66 \%)$ reconocen la necesidad de un cambio en la educación en valores; un mayor conocimiento y cumplimiento del 
Percepción de los expertos de la necesidad de la formación del profesorado en convivencia

reglamento; una mayor implicación de los agentes de la comunidad, principalmente del profesorado y las familias; y la indisciplina escolar.

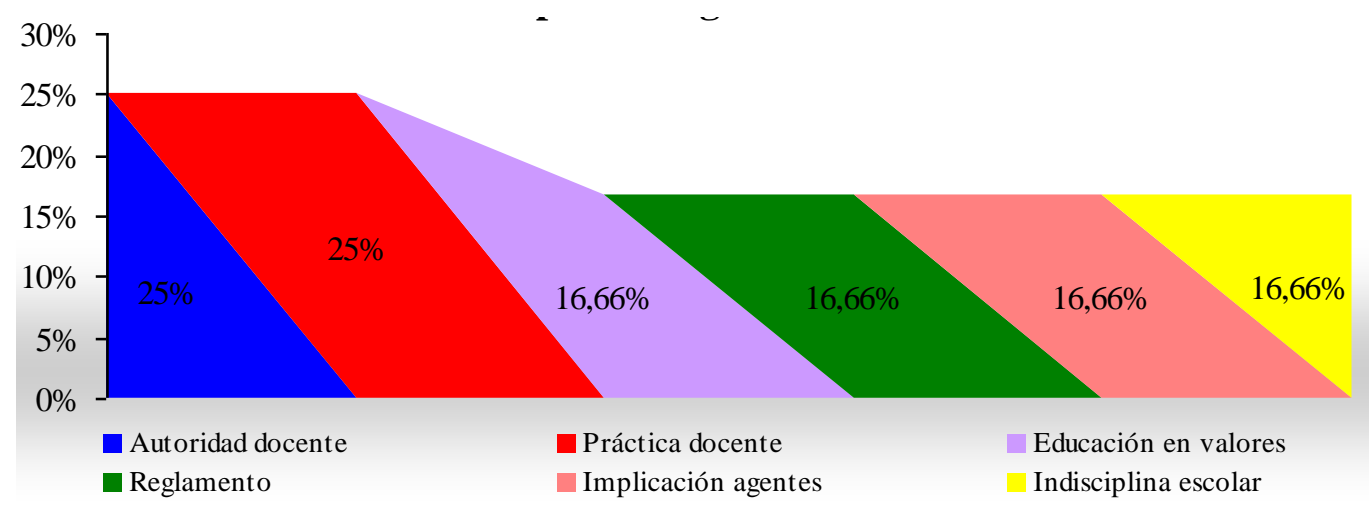

Gráfico 7. Aspectos Negativos en Secundaria

No obstante es necesario al mismo tiempo, reforzar aspectos positivos tanto en primaria como en secundaria. En esta caso el (50\%) de los expertos consideran que en educación primaria es necesario reforzar los valores de socialización, respeto, tolerancia y convivencia democrática, así como el clima escolar adecuado para desarrollar unas relaciones personales satisfactorias y un clima de trabajo que propicie el aprendizaje integral del alumnado. El $41,66 \%$ de los expertos consideran imprescindible reforzar la atención al alumnado y las familias resaltando la cercanía del profesor al alumnado, así como su predisposición a la ayuda. El 33,33\% consideran necesario fortalecer el trato y reconocimiento del docente fortaleciendo su satisfacción personal y profesional mediante la motivación, así como la percepción de la convivencia positivamente. Sin embargo, el $(16,66 \%)$ se centran en la normativa, y tan solo el $(8,33 \%)$ consideran necesario centrarse en la práctica de una autoridad auctorita.

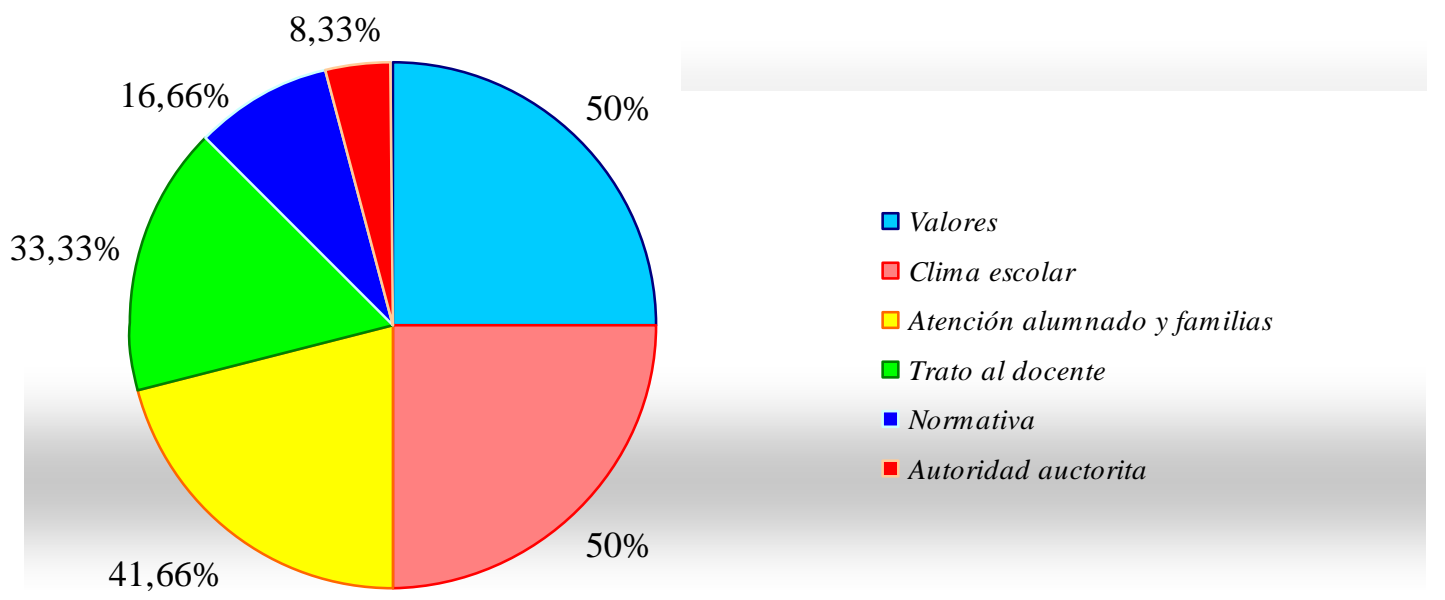

Gráfico 8. Aspectos Positivos en Primaria 
En secundaria, los expertos estiman necesario reforzar la tarea institucional así como la práctica docente (33,33\%), resaltando el trabajo en equipo y la diversidad de servicios que se ofrecen desde la institución como la mediación y la orientación educativa. El 25\% consideran imprescindible reforzar el clima de centro, el $16,66 \%$ la educación en valores democráticos y la normativa, y tan solo el 8,33\% reconocen la autoridad docente desde un modelo auctoritas como un aspecto positivo que se deba consolidar.

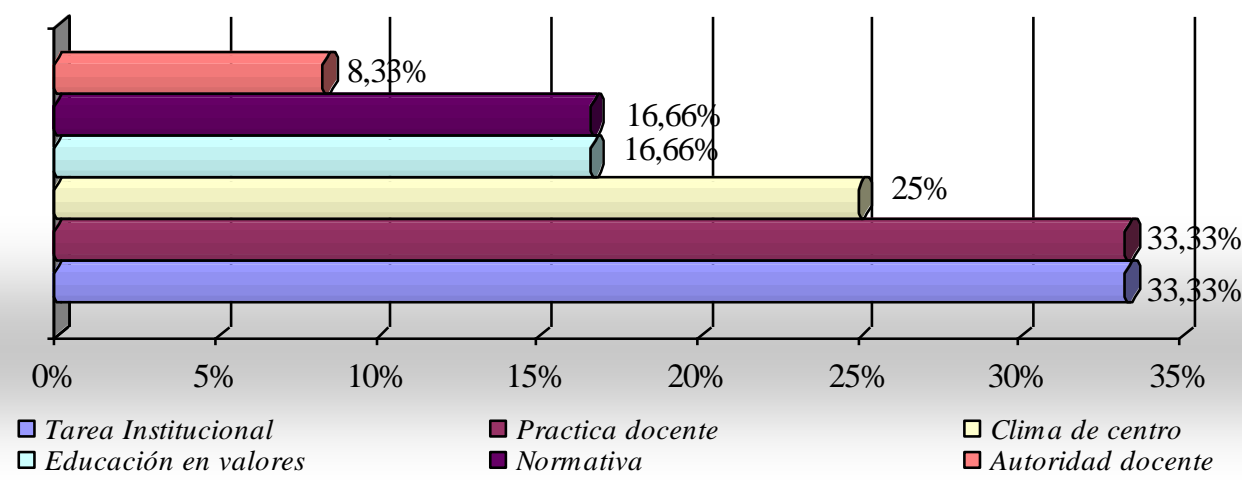

Gráfico 9. Aspectos Positivos en Secundaria

Respecto al tipo de conflictos, el acoso escolar es según los expertos el que más se produce en los centros escolares (75\%), seguido muy de cerca por la violencia física $(66,66 \%)$ y verbal $(58,33 \%)$, mientras que la discriminación y los conflictos de rendimiento y la práctica docente son reconocidos por el $(33,33 \%)$. Sin embargo son menos valorados los conflictos relacionados con el vandalismo y los daños materiales (25\%), además de los problemas entre profesores $(16,66 \%)$ y la violencia psicológica (8,33\%). Estos datos nos permiten enumerar los tipos de conflictos que pueden producirse en el aula o en el centro, y del mismo modo, dar respuesta a uno de los objetivos de nuestra investigación.

En la gráfica 10 exponemos la clasificación resultante de las respuestas obtenidas.

Uno de los principales propósitos de las investigaciones enfocadas a conocer el estado de la convivencia en las instituciones educativas y que además se recoge en nuestro estudio, es determinar qué factores son los instigadores de conflictos escolares, considerando en palabras de Hernández (2004) que es un fenómeno multicausal. Por el contrario los resultados del panel de expertos evidencian que el $(91,66 \%)$ las causas se centran principalmente en los factores escolares (la carencia de unas normas de convivencia, la mala gestión del centro, el exceso 
Percepción de los expertos de la necesidad de la formación del profesorado en convivencia

de horas lectivas que desemboca en desinterés y desmotivación en el alumnado, el estilo pedagógico del docente impositivo, dialogante o permisivo, así como la carencia de formación docente, traducida en escasez de conocimientos y competencias del equipo docente para la gestión de conflictos).
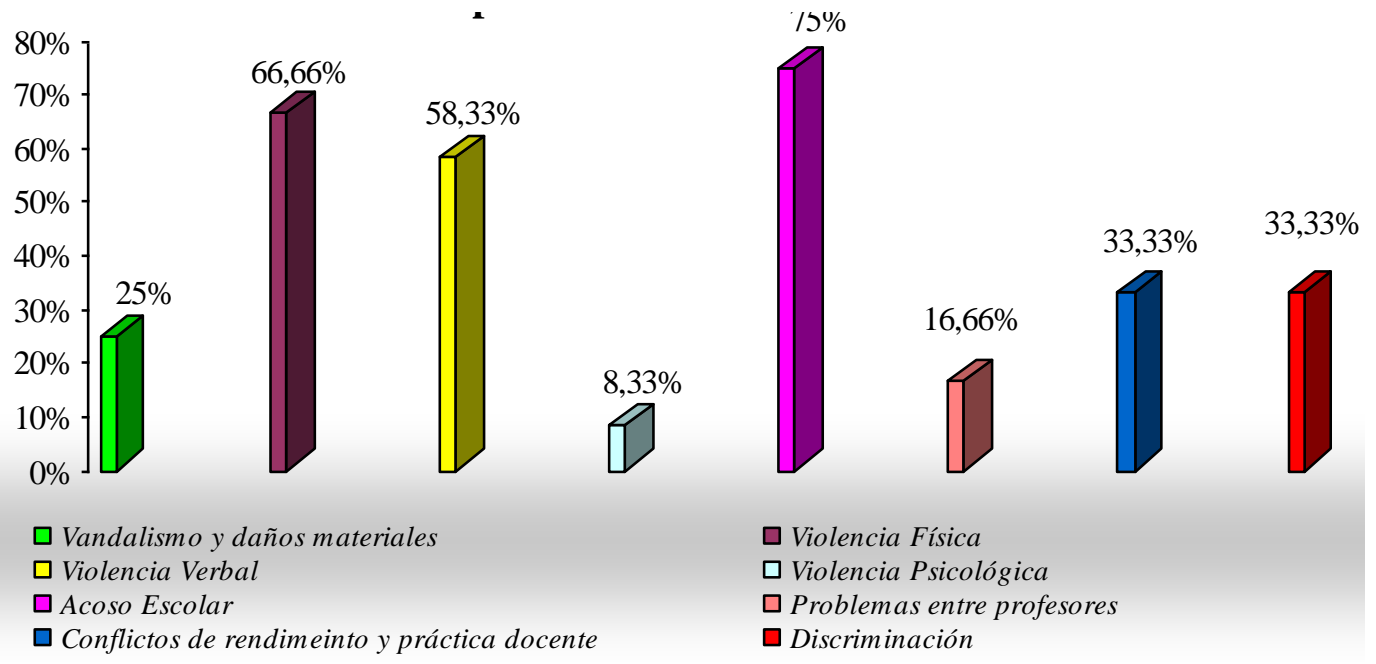

Gráfico 10. Tipos de Conflictos

En segundo lugar, el $66,66 \%$ de los expertos aluden a los factores familiares como la carencia de normas, ambientes desestructurados, despreocupación por el menor, predominio de la violencia en el ámbito familiar, elevado grado de permisividad y escasas estrategias por parte de los padres para gestionar los conflictos. No obstante, el $(58,33 \%)$ reconocen como uno de los principales instigadores de los conflictos escolares, los factores sociales, especialmente los medios de comunicación y ambientes violentos en el entorno comunitario de los menores. Sin embargo y en menor medida se detectan otros factores como los individuales y emocionales de los menores $(41,66 \%)$ y factores culturales $(8,33 \%)$.

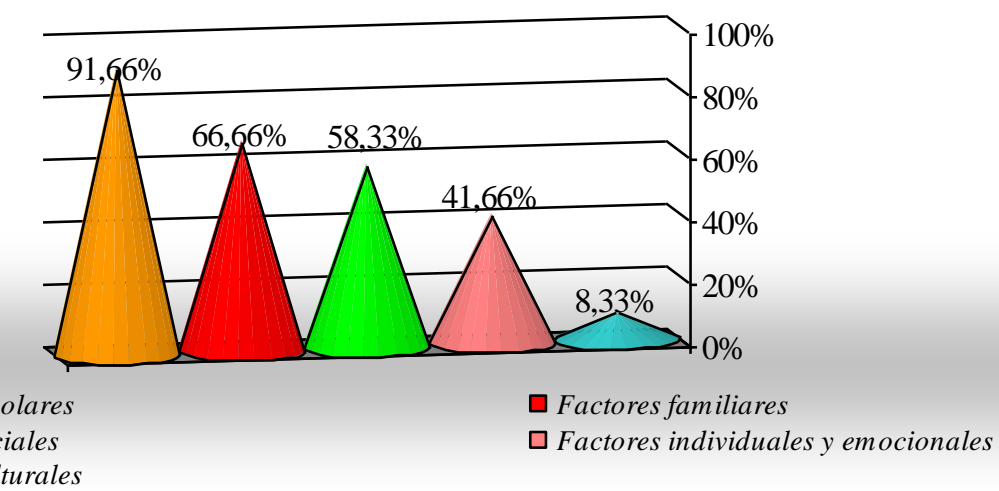

$\square$ Factores sociales

$\square$ Factores individuales y emocionales

Gráfico 11. Factores 
Por último, los expertos consideran que una convivencia protegida por conflictos escolares conlleva unas consecuencias en el deterioro instructivo (83\%) como la pérdida del rumbo en educación tanto de docentes como educando que deriva en el bajo rendimiento académico, desmotivación y absentismo y abandono escolar. Del mismo modo, el $75 \%$ de los expertos creen que afecta directamente a las relaciones entre los agentes de la comunidad educativa empobreciendo las relaciones sociales, fomentando el malestar entre docentes y alumnos, y aumentando las situaciones de aislamiento social, desconfianza y evitación entre alumnos. Sin embargo el $(33,33 \%)$ de expertos resaltan las consecuencias a nivel de centro, centradas en el deterioro de las instalaciones y del ambiente del centro, fomentando la sensación de inseguridad entre los miembros de la comunidad. Tan solo el $(16,66 \%)$ se refieren a los desajustes psicológicos y físicos que se puedan producir. Estos datos dan respuesta a otra de las dimensiones de nuestra investigación con la que se trataba de conocer que efectos o consecuencias tiene una convivencia con conflictos en los centros educativos.

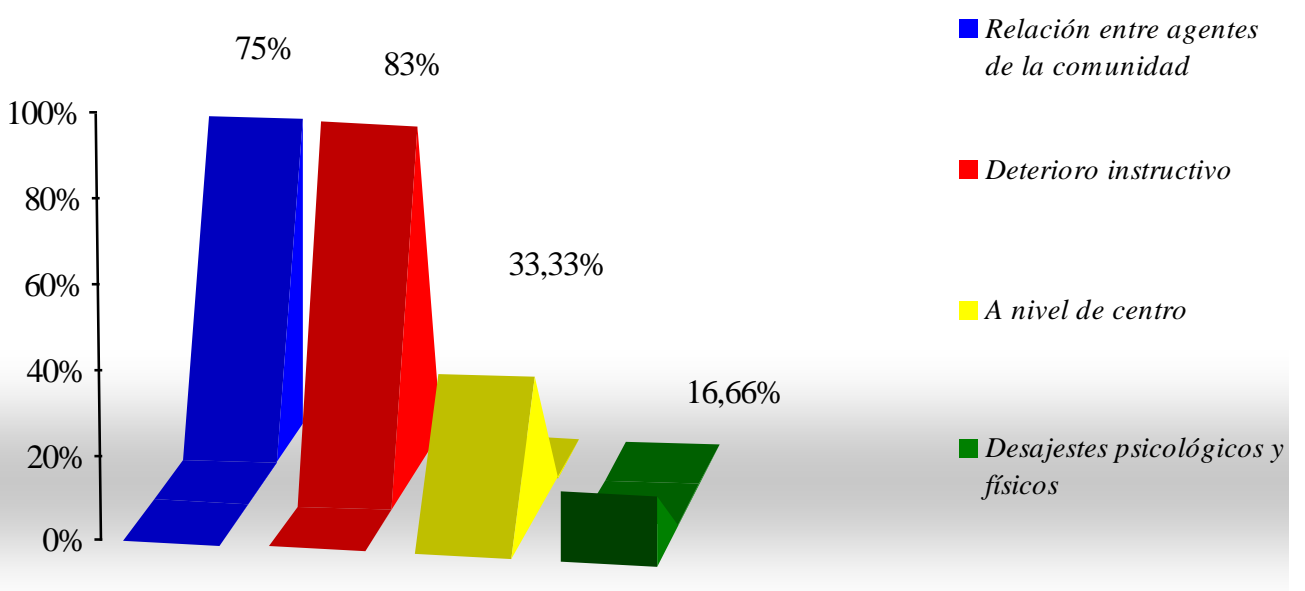

Gráfico 12. Efectos y Consecuencias

\section{CONCLUSIONES}

Los resultados extraídos del análisis del instrumento no sólo nos acercan a la realidad de los centros educativos y a la percepción del profesorado sobre convivencia, sino que demuestran la necesidad de una formación adaptada a sus necesidades pedagógicas y que desde las instituciones reclaman para mejorar sus competencias en la gestión de la convivencia. A continuación se exponen las conclusiones que hemos extraído de este trabajo: 
Percepción de los expertos de la necesidad de la formación del profesorado en convivencia

En primer lugar resaltar, la importancia que los expertos otorgan a la formación del profesorado en competencias conductuales, actitudinales y cognitivas. Sin embargo consideran de más relevancia centrar la formación en competencias conductuales, concretamente en la gestión docente, en especial, como intervenir ante problemas de convivencia y la capacidad de aplicar técnicas de gestión creativas e inclusivas, basada en el trabajo en equipo, debates, la dramatización, el uso de la literatura infantil y juvenil y el trabajo cooperativo.

En segundo lugar destacar la escasa importancia atribuida la normativa sobre convivencia escolar, en las competencias formativas, en los aspectos positivos y negativos en Primaria y en los negativos de Secundaria. A través de estos datos denotamos el déficit de interés sobre las normas de convivencia establecidas en el centro, y por ende la falta de información al respecto, datos que se confirman en el estudio realizado por (Álvarez, Rodríguez, González, Núñez y Álvarez, 2010). No obstante la normativa debería considerarse un aspecto relevante en la formación docente, que favorezca el conocimiento sobre la situación de la convivencia, sirva de fundamento para la prevención e intervención en casos de conflictos, y favorezca la implicación y participación del alumnado en la elaboración de la normativa del aula a través de la negociación.

En tercer lugar y haciendo referencia a las medidas, otorgan más relevancia a las efectuadas desde el propio centro como planes de convivencia, programas de prevención, acción tutorial, etc., que a las medidas destinadas al profesor y al alumnado. Lo mismo sucede con las estrategias que deben poseer los docentes, concediendo en este caso, mayor importancia a las estrategias de intervención que las de prevención, especialmente a la mediación, las terapias realistas, el trabajo individualizado y el trabajo cooperativo, la transmisión de valores, el control de alumnos disruptivos y el trabajo desde la acción tutorial No obstante, los expertos consideran la necesidad de aplicar estrategias preventivas, en forma de propuestas pedagógicas pues facilitan al alumnado una educación integral sobre la gestión positiva de conflictos (Boqué, 2007) que favorezcan las relaciones interpersonales y por ende, la convivencia de la institución educativa.

En cuarto lugar, resaltar la importancia concedida por los expertos al acoso escolar, la violencia física y verbal, como conflictos más habituales en los centros educativos. Lo mismo sucede con los factores familiares, escolares y sociales como instigadores de los problemas de convivencia y que según los expertos son consecuencia del deterioro instructivo y el desgaste en las relaciones interpersonales. 
Para concluir, acentuar la necesidad de los expertos por hacer constancia de aquellos aspectos que estiman indispensables para la mejora de la convivencia y por lo tanto se deben reforzar, como la educación en valores, el clima escolar y la atención al alumnado y las familias en primaria, y la tarea institucional y la práctica docente en secundaria. Lo mismo sucede con otros aspectos que deben transformarse por ser contraproducentes para unas relaciones positivas, como los comportamientos disruptivos y la escasa implicación de las familias en primaria, y la autoridad y práctica docente en secundaria.

\section{REFERENCIAS BIBLIOGRÁFICAS}

Álvarez, D; Rodríguez, C; González, P; Núñez, J.C y Álvarez, L (2010). La formación de los futuros docentes frente a la violencia escolar. Revista de Psicodidáctica, 15 (1), 35-56.

Armas, M (2007). Prevención e intervención ante problemas de conducta. Estrategias para centros educativos y familias. Gestión, Madrid.

Astigarraga, E (2007). El método Delphi. Universidad de Deusto. San Sebastián.

http://www.unalmed.edu.co/ poboyca/documentos/document os 1/documentos-

Juan\%20Diego/PInaifi_Cuencas_Pregrado/Sept_29/Metodo_delph i.pdf

Boqué, C (2007). Prevención de conflictos y mediación escolar. Idea 4 , 60-66.

http://www.doredin.mec.es/documentos/00620073000913.pdf

Castillo, A.M (2002). Técnica Delfo: La opinión de los expertos y su aportación a la toma de decisiones en la educación superior. Cuaderno de investigación en la Educación, 18.

Caurcel, M.J (2005). Capacitación profesional y necesidades formativas percibidas por los futuros educadores para abordar el maltrato entre iguales. $V$ Congreso Internacional Virtual de Educación.

Chinchurreta, M (2006). El acoso escolar y la violencia en el deporte. Posible relación. Soluciones desde la educación física. Revista digital Investigación y Educación, 22.

Comisión Europea (2005). Panel de expertos. Recuperado el 21 de Marzo de 2013

http://ec.europa.eu/europeaid/evaluation/methodology/tools/to o_pan_whe_es.htm

Del Rey, R y Ortega, R (2001). Programas para la prevención de la violencia escolar en España: La respuesta de las comunidades autónomas. Revista interuniversitaria de Formación del profesorado, 41, 133-145. 
Percepción de los expertos de la necesidad de la formación del profesorado en convivencia

Delors, J (1996). La educación encierra un tesoro. Informe a la UNESCO de la Comisión Internacional sobre la Educación para el Siglo XXI. Santillana. UNESCO.

Fernández- Ballesteros, R (1995). Evaluación de programas: una guía práctica en ámbitos sociales, educativos y de salud. Madrid: Síntesis.

Fernández, L (2006). ¿Cómo analizar datos cualitativos?. Butlleti LaRecerca, 7.http://www.ub.edu/ice/recerca/pdf/ficha7-cast.pdf

García, T (2003). El cuestionario como instrumento de investigación/evaluación. Ełapas del proceso investigador: Instrumentación. Almendralejo. Recuperado el 21 de Marzo de 2013. http://www.univsantana.com/sociologia/El_Cuestionario.pdf

Jares, $X$ (2006). Conflicto y convivencia en los centros educativos de secundaria. Revista de Educación, 339, 467-491.

Landeta, J (1999). Análisis de datos. Etapa exploratoria. Madrid. Pirámide.

Landeta, J. (1999). El método Delphi. Una técnica de previsión para la incertidumbre. Barcelona: Ariel.

Martínez, E (2003). La técnica Delphi como estrategia de consulta a los implicados en la evaluación de programas. Revista de investigación educativa, 21 (2), 449-463.

Narejo, N y Salazar, M. (2002). "Vías para abordar los conflictos en el aula". Revista Electrónica Interuniversitaria de Formación del Profesorado, 5(4).

http://www.aufop.com/aufop/revistas/arta/digital/109/833

Ortega, R. (2006) La convivencia: un modelo para de prevención de la violencia. En Moreno, A. (Eds.), La convivencia en las aulas: problemas y soluciones, 29-48. Volumen de las Intervenciones del Congreso: La convivencia en las aulas: Problemas y soluciones, (2005) España: Ministerio de Educación y Ciencia.

Ortega, R., Fernández, I. y Del Rey, R. (2002). Abordar la Violencia en las Escuelas: Un Informe de España.

Rodríguez, R (2007). Los planes de convivencia como herramientas para prevenir los conflictos escolares. Jornadas Europeas sobre convivencia escolar.

Ruiz Olabuénaga, J. I e Ispuzua, M.A (1989). La descodificación de la vida cotidiana. Bilbao: Universidad de Deusto.

Tobón, S. (2008). Centro de Investigación en Formación y Evaluación. Recuperado el 10 de diciembre de 2008, de: Principales enfoques de las competencias en educación superior: http://www.cife.ws

Uruñuela, P.M (2012) La formación del profesorado en convivencia. Revista digital de educación y formación del profesorado.

Vásquez, A (2010). Competencias cognitivas en la Educación Superior. Revista Electrónica de Desarrollo de Competencias (REDEC), 6 (2), 34-64. 
http://www.educandus.cl/ojs/index.php/fcompetencias/article/viewFile $/ 79 / 84$

ANEXO I: TABLA DE CODIFICACIÓN DE LOS PANELES.

\begin{tabular}{|l|l|l|l|l|l|}
\hline \multicolumn{2}{|l|}{ ASPECTOS POSITIVOS } & UNID. DE SIG & NUM. & $\%$ \\
\hline CATEGORÍAS & CODIGO & MUESTREO & UNI & & \\
\hline \multirow{4}{*}{} & & & & & \\
\cline { 3 - 4 } & & & & \\
\cline { 3 - 4 } & & & & \\
\cline { 3 - 4 } & & & & & \\
\cline { 3 - 4 } & & & & & \\
\end{tabular}

\section{ANEXO II: UNIDADES DE INFORMACIÓN}

\begin{tabular}{|c|c|c|c|c|c|c|c|c|c|}
\hline \multicolumn{2}{|c|}{ PREGUNTA } & \multicolumn{8}{|c|}{ CATEGORÍAS } \\
\hline \multirow{4}{*}{$\begin{array}{l}\mathrm{P} \\
\mathrm{r} \\
\mathrm{e} \\
\mathrm{g} \\
\mathrm{u} \\
\mathrm{n} \\
\mathrm{t} \\
\mathrm{a} \\
\mathrm{l}\end{array}$} & $\begin{array}{l}\text { Aspectos } \\
\text { positivos de } \\
\text { primaria }\end{array}$ & $\begin{array}{l}\text { Educación } \\
\text { en valores }\end{array}$ & Clima Escolar & $\begin{array}{l}\text { Autoridad } \\
\text { auctoritas }\end{array}$ & $\begin{array}{l}\text { Normas de } \\
\text { convivencia }\end{array}$ & $\begin{array}{l}\text { Atenci } \\
\text { ón al } \\
\text { alumn } \\
\text { ado y } \\
\text { familia }\end{array}$ & $\begin{array}{l}\text { Tra } \\
\text { pro }\end{array}$ & al & \\
\hline & $\begin{array}{l}\text { Aspectos } \\
\text { negativos } \\
\text { de primaria }\end{array}$ & $\begin{array}{l}\text { Carencia } \\
\text { de } \\
\text { Educación } \\
\text { en valores }\end{array}$ & Normativa & $\begin{array}{l}\text { Comporta } \\
\text { miento } \\
\text { disruptivos }\end{array}$ & $\begin{array}{l}\text { Implicación } \\
\text { familiar y } \\
\text { atención al } \\
\text { alumnado }\end{array}$ & \multicolumn{4}{|c|}{ Práctica docente } \\
\hline & $\begin{array}{l}\text { Aspectos } \\
\text { positivos de } \\
\text { secundaria }\end{array}$ & $\begin{array}{l}\text { Educación } \\
\text { en valores }\end{array}$ & Reglamento & $\begin{array}{l}\text { Clima del } \\
\text { centro }\end{array}$ & $\begin{array}{l}\text { Autoridad } \\
\text { docente }\end{array}$ & \multicolumn{4}{|c|}{$\begin{array}{l}\text { Institución y } \\
\text { práctica } \\
\text { docente }\end{array}$} \\
\hline & $\begin{array}{l}\text { Aspectos } \\
\text { negativos } \\
\text { de } \\
\text { secundaria }\end{array}$ & $\begin{array}{l}\text { Educación } \\
\text { en valores }\end{array}$ & $\begin{array}{l}\text { Reglamento } \\
\text { secundaria }\end{array}$ & Implicación & $\begin{array}{l}\text { Práctica } \\
\text { pedagógica } \\
\text { y atención al } \\
\text { alumnado }\end{array}$ & $\begin{array}{l}\text { Autori } \\
\text { dad }\end{array}$ & Ind & ciplina & \\
\hline $\begin{array}{l}P \\
r \\
e \\
g \\
u \\
n \\
\dagger \\
a \\
2\end{array}$ & $\begin{array}{l}\text { Tipo de } \\
\text { conflictos }\end{array}$ & $\begin{array}{l}\text { Vandalismo } \\
\text { y daños } \\
\text { materiales }\end{array}$ & $\begin{array}{l}\text { Violencia } \\
\text { física }\end{array}$ & $\begin{array}{l}\text { Violencia } \\
\text { verbal }\end{array}$ & $\begin{array}{l}\text { Violencia } \\
\text { psicológica }\end{array}$ & $\begin{array}{l}\text { Acoso } \\
\text { escola } \\
r\end{array}$ & $\begin{array}{l}\mathrm{Pr} \\
\mathrm{O} \\
\mathrm{bl} \\
\mathrm{e} \\
\mathrm{m} \\
\mathrm{as} \\
\mathrm{e} \\
\mathrm{nt} \\
\mathrm{re} \\
\mathrm{pr} \\
\text { of } \\
\text { es } \\
\text { or } \\
\text { es }\end{array}$ & $\begin{array}{l}\text { Conflic } \\
\text { tos de } \\
\text { rendim } \\
\text { iento y } \\
\text { prácti } \\
\text { ca } \\
\text { docen } \\
\text { te }\end{array}$ & $\begin{array}{l}\mathrm{Di} \\
\mathrm{sc} \\
\mathrm{ri} \\
\mathrm{mi} \\
\mathrm{n} \\
\mathrm{a} \\
\mathrm{ci} \\
\mathrm{o} \\
\mathrm{n}\end{array}$ \\
\hline $\begin{array}{l}P \\
r \\
e \\
g \\
u \\
n \\
\dagger \\
a\end{array}$ & Factores & $\begin{array}{l}\text { Factores } \\
\text { escolares }\end{array}$ & $\begin{array}{l}\text { Factores } \\
\text { sociales }\end{array}$ & $\begin{array}{l}\text { Factores } \\
\text { familiares }\end{array}$ & $\begin{array}{l}\text { Factores } \\
\text { individuales y } \\
\text { emocionales }\end{array}$ & \multicolumn{4}{|c|}{ Factores culturales } \\
\hline
\end{tabular}


Percepción de los expertos de la necesidad de la formación del profesorado en convivencia

\begin{tabular}{|c|c|c|c|c|c|}
\hline 3 & & & & & \\
\hline $\begin{array}{l}P \\
r \\
\text { r } \\
\text { e } \\
g \\
u \\
n \\
\dagger \\
\text { a } \\
4\end{array}$ & $\begin{array}{l}\text { Efectos y } \\
\text { consecuen } \\
\text { cias }\end{array}$ & $\begin{array}{l}\text { Relaciones } \\
\text { entre } \\
\text { agentes de } \\
\text { la } \\
\text { comunidad }\end{array}$ & $\begin{array}{l}\text { Deterioro } \\
\text { instructivo }\end{array}$ & $\begin{array}{l}\text { A nivel de } \\
\text { centro }\end{array}$ & Desajustes psicologicos y físicos \\
\hline $\begin{array}{l}\mathrm{P} \\
\mathrm{r} \\
\mathrm{e} \\
\mathrm{g} \\
\mathrm{u} \\
\mathrm{n} \\
\dagger \\
\mathrm{a} \\
5\end{array}$ & Medidas & $\begin{array}{l}\text { Dirigidas al } \\
\text { alumno }\end{array}$ & $\begin{array}{l}\text { Dirigidas al } \\
\text { docente }\end{array}$ & & \\
\hline $\begin{array}{l}P \\
r \\
e \\
g \\
u \\
n \\
t \\
a \\
b\end{array}$ & Estrategias & $\begin{array}{l}\text { Estrategias } \\
\text { de } \\
\text { intervenció } \\
n\end{array}$ & & & \\
\hline $\begin{array}{l}P \\
r \\
e\end{array}$ & $\begin{array}{l}\text { Competen } \\
\text { cias } \\
\text { cognitivas }\end{array}$ & $\begin{array}{l}\text { Causas del } \\
\text { conflicto }\end{array}$ & $\begin{array}{l}\text { Estrategias y } \\
\text { métodos }\end{array}$ & Normativa & \\
\hline $\begin{array}{l}g \\
u \\
n \\
t\end{array}$ & $\begin{array}{l}\text { Competen } \\
\text { cias } \\
\text { conductual } \\
\text { es }\end{array}$ & $\begin{array}{l}\text { Gestión } \\
\text { docente }\end{array}$ & Valores & Normas & \\
\hline $\begin{array}{l}a \\
7\end{array}$ & $\begin{array}{l}\text { Competen } \\
\text { cias } \\
\text { actitudinal } \\
\text { es } \\
\end{array}$ & $\begin{array}{l}\text { Actitudes } \\
\text { relacionales }\end{array}$ & Actitudes ed & ativas & \\
\hline
\end{tabular}




\section{Sobre las autoras:}

\section{Antonia Penalva López}

antonia.penalva@um.es

Diplomada en Educación social, licenciada en Pedagogía, Máster en investigación e innovación en educación infantil y educación primaria, doctoranda en la Universidad de Murcia. Su producción científica está centrada en la gestión del aula, concretamente en las competencias del docente, y en la formación del profesorado en convivencia escolar y gestión de conflictos.

\section{Mª́ngeles Hernández Prados}

mangeles@um.es

Doctora en Pedagogía y profesora en el Departamento de Teoría e Historia de la Educación en la Facultad de Educación de la Universidad de Murcia. Miembro del Grupo de investigación consolidado "Actitudes y valores". Participa en diversos cursos de formación familiar y del profesorado. Su producción científica se concentra en el campo de la educación en valores, la convivencia escolar, educación y formación familiar, así como el planteamiento ético de las TIC.

\section{Catalina Guerrero Romera} cgromera@um.es

Profesora de la Facultad de Educación de la Universidad de Murcia. Coordinadora de Innovación del Vicerrectorado de Formación e Innovación de dicha Universidad. Consultora de Formación y Desarrollo Organizativo. Sus líneas de investigación actuales, algunas publicadas en diversas revistas de especialización científica, están relacionadas con la Formación, Evaluación e Innovación en las Organizaciones, la Gestión del Conocimiento y el Aprendizaje Organizacional y la Integración de Colectivos Vulnerables a través de la Formación y la Orientación.

\section{Para citar este artículo:}

Penalva, A.; Hernández, M.A. y Guerrero, C. (2014). Percepción de los expertos de la necesidad de la formación del profesorado en convivencia. Revista Fuentes, 15, Junio, pp. 281-304. [Fecha de consulta: dd/mm/aaaa]. http://www.revistafuentes.es/ D.O.I.: http://dx.doi.org/10.12795/revistafuentes.2014.i15.13 
Percepción de los expertos de la necesidad de la formación del profesorado en convivencia 